\title{
Balanced factor congruences of double MS-algebras
}

\author{
Abd El-Mohsen Badawy
}

Correspondence:

abdel-mohsen.mohamed@science. tanta.edu.eg

Department of Mathematics,

Faculty of Science, Tanta University,

Tanta, Egypt

\begin{abstract}
In this paper, we introduce the concepts of Stone elements, central elements and Birkhoof central elements of a double MS-algebra and study their related properties. We observe that the center $C(L)$ of a double MS -algebra $L$ is precisely the Birkhoof center $B C(L)$ of $L$. A complete description of factor congruences on a double MS-algebra is given by means of the central elements. A characterization of balanced factor congruences of double MS-algebra is obtained. A one-to-one correspondence between the class of all balanced factor congruences of a double MS-algebra $L$ and the central elements of $L$ is obtained.
\end{abstract}

Keywords: De Morgan algebra, MS-algebra, Double MS-algebra, Factor congruences, Balanced factor congruences

2010 Mathematics Subject Classification: Primary 06D30, Secondary 06D15

\section{Introduction}

Blyth and Varlet [1] introduced MS-algebras as a generalization of both de Morgan algebras and Stone algebras. Blyth and Varlet [2] characterized the subvarieties of the class MS of all MS-algebras. Badawy, Guffova, and Haviar [3] introduced and characterized the class of principal $M S$-algebras and the class of decomposable $M S$-algebras by means of triples. Badawy [4] introduced and studied many properties of $d_{L}$-filters of principal $M S$ algebras. Also, Badawy and El-Fawal [5] considered homomorphisms and subalgebras of decomposable $M S$-algebras.

Blyth and Varlet [6] introduced the class of double $M S$-algebras and showed that every de Morgan algebra $M$ can be represented non-trivially as the skeleton of the double $M S$ algebra $M^{[2]}=\{(a, b) \in M \times M: a \leq b\}$. The class of double $M S$-algebras satisfying the complement property has been introduced by Congwen [7]. Haviar [8] studied affine complete of double $M S$-algebras from the class $\mathbf{K}_{2}$, of all double $K$-algebras. Wang [9] introduced the notion of congruence pairs of double $K_{2}$-algebras. Recently, Badawy [10] introduced and constructed the class of double $M S$-algebras satisfying the generalized complement property that is containing the class of double $M S$-algebras satisfying the complement property.

In this paper, we introduce the notion of Stone elements in double $M S$-algebras. Then, we prove that the set of Stone elements of a double $M S$-algebra $L$ forms the greatest Stone subalgebra of $L$. We introduce the concept of central elements of a double $M S$-algebra $L$

(c) The Author(s). 2019 Open Access This article is distributed under the terms of the Creative Commons Attribution 4.0 International License (http://creativecommons.org/licenses/by/4.0/), which permits unrestricted use, distribution, and reproduction in any medium, provided you give appropriate credit to the original author(s) and the source, provide a link to the Creative Commons license, and indicate if changes were made. 
and we show that the set $C(L)$ of all central elements forms the greatest Boolean subalgebra of $L$. For a principal ideal (a] (filter $[a)$ ) of a double $M S$-algebra $\left(L ;^{\circ},+\right)$, it is observed that a relativized algebra $(a]_{L}=\left((a] ; \vee, \wedge,{ }^{\circ} a,{ }^{+} a, 0, a\right)\left([a)_{L}=\left([a) ; \vee, \wedge,{ }^{\circ}{ }^{\circ},{ }^{+a}, a, 1\right)\right)$ is a double $M S$-algebra if and only if $a$ is a central element of $L$, where $x^{\circ a}=x^{\circ} \wedge a$ and $x^{+a}=x^{+} \wedge a\left(x^{\circ a}=x^{\circ} \vee a\right.$ and $\left.x^{+a}=x^{+} \vee a\right)$. Also, we introduce the Birkhoof center of a double $M S$-algebra, then we showed that the Birokhoof center of a double $M S$-algebra $L$ can be identified with the center of $L$. Factor congruences of a double $M S$-algebra are investigated by means of central elements. Finally, we study and characterize balanced factor congruences of a double $M S$-algebra. There is one-to-one correspondence between the class of balanced factor congruences of a double $M S$-algebra $L$ and the center $C(L)$ of $L$.

\section{Preliminaries}

In this section, some definitions and results were introduced in $[1,2,6,11,12]$.

A de Morgan algebra is an algebra $\left(L ; \vee, \wedge^{-}, 0,1\right)$ of type $(2,2,1,0,0)$ where $(L ; \vee, \wedge, 0,1)$ is a bounded distributive lattice and ${ }^{-}$the unary operation of involution satisfies:

$\overline{\bar{x}}=x, \overline{(x \vee y)}=\bar{x} \wedge \bar{y}, \overline{(x \wedge y)}=\bar{x} \vee \bar{y}$.

An $M S$-algebra is an algebra $\left(L ; \vee, \wedge,{ }^{\circ}, 0,1\right)$ of type $(2,2,1,0,0)$ where $(L ; \vee, \wedge, 0,1)$ is a bounded distributive lattice and a unary operation ${ }^{\circ}$ satisfies:

$x \leq x^{\circ \circ},(x \wedge y)^{\circ}=x^{\circ} \vee y^{\circ}, 1^{\circ}=0$.

The basic properties of $M S$-algebras are given in the following theorem.

Theorem 1 (Blyth and Varlet [6]) For any two elements $a, b$ of an MS-algebra L, we have (1) $0^{\circ \circ}=0$ and $1^{\circ \circ}=1$,

(2) $a \leq b \Rightarrow b^{\circ} \leq a^{\circ}$,

(3) $a^{\circ 00}=a^{\circ}$,

(4) $a^{0000}=a^{\circ 0}$,

(5) $(a \vee b)^{\circ}=a^{\circ} \wedge b^{\circ}$,

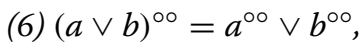

(7) $(a \wedge b)^{\circ \circ}=a^{\circ \circ} \wedge b^{\circ \circ}$.

A dual $M S$-algebra is an algebra $\left(L ; \vee, \wedge^{+}, 0,1\right)$ of type $(2,2,1,0,0)$ where $(L ; \vee, \wedge, 0,1)$ is a bounded distributive lattice and the unary operation ${ }^{+}$satisfies:

$x \geq x^{++},(x \wedge y)^{+}=x^{+} \vee y^{+}, 0^{+}=1$.

Proposition 1 For any two elements $a, b$ of a dual MS-algebra $\left(L_{;}^{+}\right)$, we have

(1) $0^{++}=0$ and $1^{++}=1$,

(2) $a \leq b \Rightarrow b^{+} \leq a^{+}$,

(3) $a^{+++}=a^{+}$,

(4) $a^{++++}=a^{++}$,

(5) $(a \vee b)^{+}=a^{+} \wedge b^{+}$,

(6) $(a \vee b)^{++}=a^{++} \vee b^{++}$,

(7) $(a \wedge b)^{++}=a^{++} \wedge b^{++}$.

A double $M S$-algebra is an algebra $\left(L ;^{\circ},+\right)$ such that $\left(L ;^{\circ}\right)$ is an $M S$-algebra, $\left(L ;^{+}\right)$is a dual $M S$-algebra, and the unary operations ${ }^{\circ},+$ are linked by the identities $x^{+\circ}=x^{++}$and $x^{\circ+}=x^{\circ \circ}$, for all $x \in L$. 
For any element $x$ of a double $M S$-algebra $L$, it is clear that $x^{++} \leq x^{\circ \circ}$ and consequently $x^{\circ} \leq x \leq x^{+}$.

Some subsets of a double $M S$-algebra play a significant role in the investigation, by the skeleton $L^{\circ \circ}$ of a double $M S$-algebra $L$ we mean a de Morgan algebra

$L^{\circ \circ}=\left\{x \in L: x=x^{\circ \circ}\right\}=L^{++}=\left\{x \in L: x=x^{++}\right\}=\left\{x \in L: x^{\circ}=x^{+}\right\}$.

An equivalence relation $\theta$ on a lattice $L$ is called a lattice congruence on $L$ if $(a, b) \in \theta$ and $(c, d) \in \theta$ implies $(a \vee c, b \vee d) \in \theta$ and $(a \wedge c, b \wedge d) \in \theta$.

Theorem 2 (Blyth [12]) An equivalence relation on a lattice L is a lattice congruence on $L$ if and only if $(a, b) \in \theta$ implies $(a \vee z, b \vee z) \in \theta$ and $(a \wedge z, b \wedge z) \in \theta$ for all $z \in L$.

A lattice congruence $\theta$ on a double $M S$-algebra $\left(L ;^{\circ},+\right)$ is called a congruence on $L$ if $(a, b) \in \theta$ implies $\left(a^{\circ}, b^{\circ}\right) \in \theta$ and $\left(a^{+}, b^{+}\right) \in \theta$.

We use $\nabla=L \times L$ for the universal congruence on a lattice $L$ and $\Delta=\{(a, a): a \in L\}$ for the equality congruence on $L$.

We say the congruences $\theta, \psi$ on a lattice $L$ are permutable if $\theta \circ \psi=\psi \circ \theta$, that is, $x \equiv y(\theta)$ and $y \equiv z(\psi)$ imply $x \equiv r(\psi)$ and $r \equiv z(\theta)$ for some $y, r \in L$.

\section{Center and Birkhoof center of a double $M S$-algebra}

We introduce the concept of Stone elements of a double $M S$-algebra $L$. Then, we show that the set $L_{S}$ of all Stone elements of $L$ is the greatest Stone subalgebra of $L$.

Definition 1 An element $x$ of a double MS-algebra $L$ is called a Stone element of $L$ if $x^{\circ} \vee x^{\circ \circ}=1$ and $x^{+} \wedge x^{++}=0$. Let $L_{S}$ denote the set of all Stone elements of $L$, that is, $L_{S}=\left\{x \in L: x^{\circ} \vee x^{\circ \circ}=1, x^{+} \wedge x^{++}=0\right\}$.

Definition 2 Let $L_{1}$ be a bounded sublattice of a double MS-algebra $L$. Then, $L_{1}$ is called a subalgebra of $L$ if $x^{\circ}, x^{+} \in L_{1}$ for every $x \in L_{1}$.

Definition 3 A subalgebra $L_{1}$ of a double MS-algebra $L$ is called a Stone subalgebra if $x^{\circ} \vee x^{\circ \circ}=1$ and $x^{+} \wedge x^{++}=0$, for all $x \in L_{1}$.

Proposition $2 L_{S}$ is the greatest Stone subalgebra of a double MS-algebra L.

Proof It is clear that $0,1 \in L_{S}$. Let $x, y \in L_{S}$. Then, $x^{\circ} \vee x^{\circ \circ}=1, x^{+} \wedge x^{++}=0$, $y^{\circ} \vee y^{\circ \circ}=1$, and $y^{+} \wedge y^{++}=0$. Thus, we get

$$
\begin{aligned}
(x \vee y)^{\circ} \vee(x \vee y)^{\circ \circ} & =\left(x^{\circ} \wedge y^{\circ}\right) \vee\left(x^{\circ \circ} \vee y^{\circ \circ}\right) \text { by Theorem } 1(5),(6) \\
& =\left(x^{\circ} \vee x^{\circ \circ} \vee y^{\circ \circ}\right) \wedge\left(y^{\circ} \vee x^{\circ \circ} \vee y^{\circ \circ}\right) \text { by distributivity of } L \\
& =1 \wedge 1=1 \text { as } x^{\circ} \vee x^{\circ \circ}=1, y^{\circ} \vee y^{\circ \circ}=1, \\
(x \vee y)^{+} \wedge(x \vee y)^{++} & =\left(x^{+} \wedge y^{+}\right) \wedge\left(x^{++} \vee y^{++}\right) \text {by Proposition 1(5),(6) } \\
& =\left(x^{+} \wedge y^{+} \wedge x^{++}\right) \vee\left(x^{+} \wedge y^{+} \wedge y^{++}\right) \text {by distributivity of } L \\
& =0 \vee 0=0 \text { as } x^{+} \wedge x^{++}=0, y^{+} \wedge y^{++}=0 .
\end{aligned}
$$


Then, $x \vee y \in L_{S}$. Using a similar way, we get $x \wedge y \in L_{S}$. Therefore, $\left(L_{S}, \vee, \wedge, 0,1\right)$ is a bounded distributive sublattice of $L$. Now, we prove that $x^{+} \in L_{S}$ for all $x \in L_{S}$.

$$
\begin{aligned}
x^{+\circ} \vee x^{+\circ} & =x^{++} \vee x^{+++} \text {as } x^{+\circ}=x^{++} \\
& =\left(x^{+} \wedge x^{++}\right)^{+} \text {by Proposition } 1(5) \\
& =0^{+}=1 \text { as } x^{+} \wedge x^{++}=0, \\
x^{+\circ} \wedge x^{+\circ} & =x^{++} \wedge x^{+++} \text {as } x^{+\circ}=x^{++} \\
& =x^{++} \wedge x^{+}=0 \text { by Proposition } 1(3) .
\end{aligned}
$$

Hence, $x^{+} \in L_{S}$. Similarly, we can prove that $x^{\circ} \in L_{S}$ for all $x \in L_{S}$. Therefore, $L_{S}$ is a subalgebra of a double $M S$-algebra $L$. Since $x^{\circ} \vee x^{\circ \circ}=1$ and $x^{+} \wedge x^{++}=0$ for every $x \in L_{S}$, then $L_{S}$ is a Stone subalgebra of $L$. To prove that $L_{S}$ is the greatest Stone subalgebra of $L$, let $S$ be any Stone subalgebra of $L$. Let $x \in S$. Then, $x$ is a Stone element of $L$, and hence, $x \in L_{S}$. So $S \subseteq L_{S}$ as claimed.

On the following, we introduce the notion of central elements of a double $M S$-algebra $L$ and prove that the set $C(L)$ of all central elements of $L$ is the greatest Boolean subalgebra of $L$. Also, the relationship among $L_{S}, C(L)$, and $L^{\circ \circ}$ is investigated.

Definition 4 An element a of double MS-algebra $L$ is called a central element if $x \vee x^{\circ}=$ 1 and $x \wedge x^{+}=0$. The set of all central elements of $L$ is called the center of $L$ and is denoted by $C(L)$, that is, $C(L)=\left\{x \in L: x \vee x^{\circ}=1, x \wedge x^{+}=0\right\}$.

Example 1 Consider the bounded distributive lattice L in Fig. 1. Define unary operations $\circ$, on L by

$$
b^{\circ}=x^{\circ}=a, d^{\circ}=y^{\circ}=c, 1^{\circ}=z^{\circ}=0,0^{\circ}=1, c^{\circ}=d, a^{\circ}=b
$$

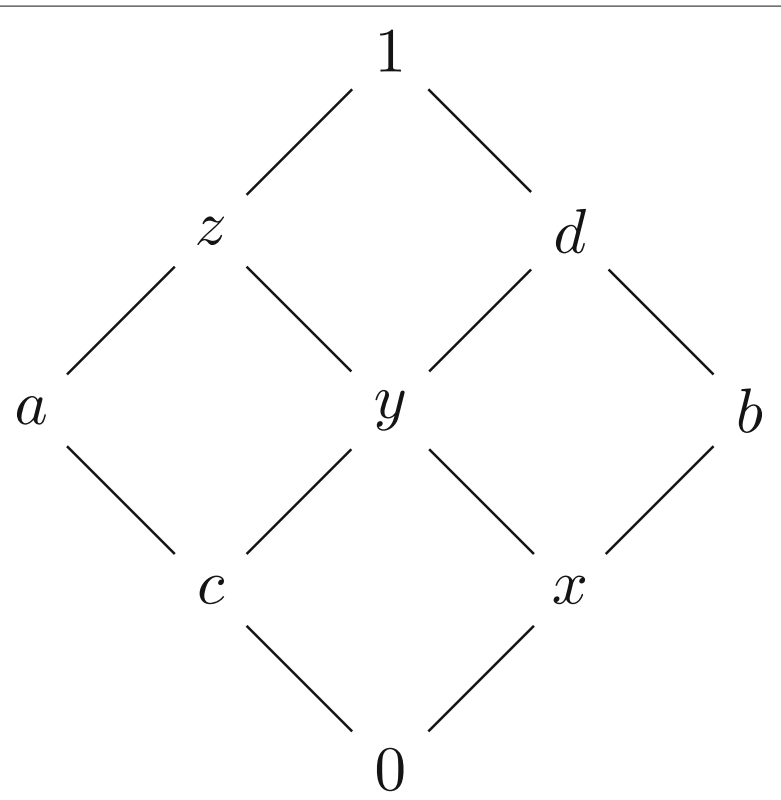

Fig. $1 L$ 
and

$$
a^{+}=z^{+}=b, c^{+}=y^{+}=d, 0^{+}=x^{+}=1, b^{+}=a, d^{+}=c, 1^{+}=0 .
$$

It is clear that $\left(L_{;^{\circ}}^{\circ},+\right)$ is a double MS-algebra. Then, $L^{\circ \circ}, L_{S}$, and $C(L)$ are given in Figs. 2, 3, and 4, respectively.

Theorem 3 Let $L$ be a double MS-algebra. Then

(1) $C(L)=L^{\circ \circ} \cap L_{S}$,

(2) $C(L)$ is the greatest Boolean subalgebra of $L, L_{S}$, and $L^{\circ \circ}$,

(3) $C(L)=C\left(L_{S}\right)=C\left(L^{\circ \circ}\right)$.

Proof (1). Let $x \in C(L)$. Then, $x \vee x^{\circ}=1$ and $x \wedge x^{+}=0$. Then

$$
\begin{aligned}
x^{++} & =x^{++} \vee 0 \\
& =x^{++} \vee\left(x \wedge x^{+}\right) \\
& =\left(x^{++} \vee x\right) \wedge\left(x^{++} \vee x^{+}\right) \text {by distributivity of } L \\
& =x \wedge\left(x^{+} \wedge x\right)^{+} \text {as } x \geq x^{++} \\
& =x \wedge 0^{+}=x \wedge 1=x .
\end{aligned}
$$

Thus, $x \in L^{\circ \circ}$. Also,

$$
\begin{aligned}
x^{++} \wedge x^{+} & =x^{++} \wedge x^{+++} \text {by Proposition } 1(3) \\
& =\left(x \wedge x^{+}\right)^{++} \text {by Proposition } 1(7) \\
& =0^{++}=0 \text { by Proposition } 1(1), \\
x^{\circ} \vee x^{\circ} & \geq x \vee x^{\circ} \text { as } x^{\circ \circ} \geq x \\
& =1 .
\end{aligned}
$$

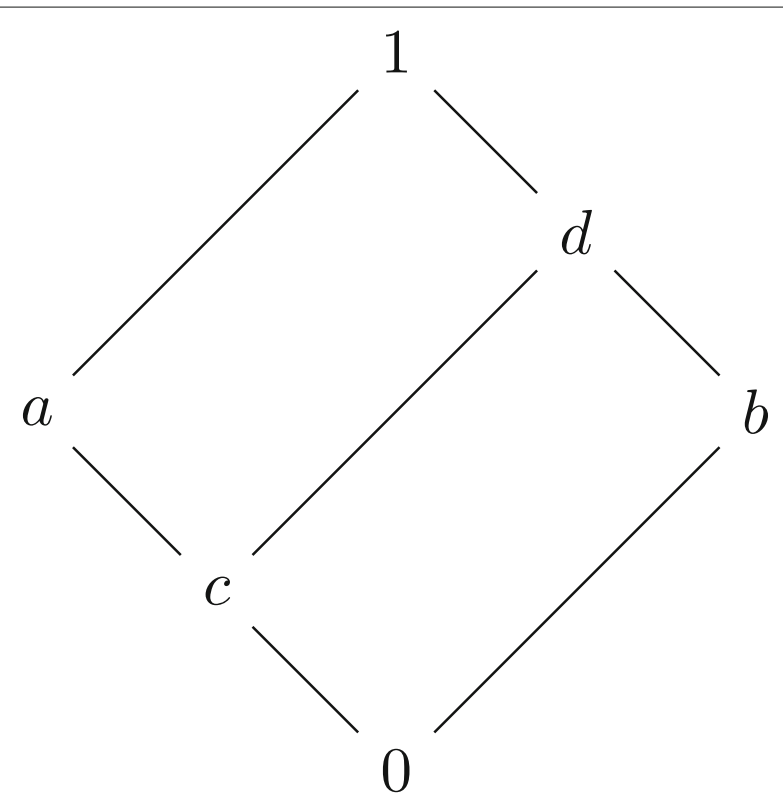

Fig. $2 L^{\circ 0}$ 


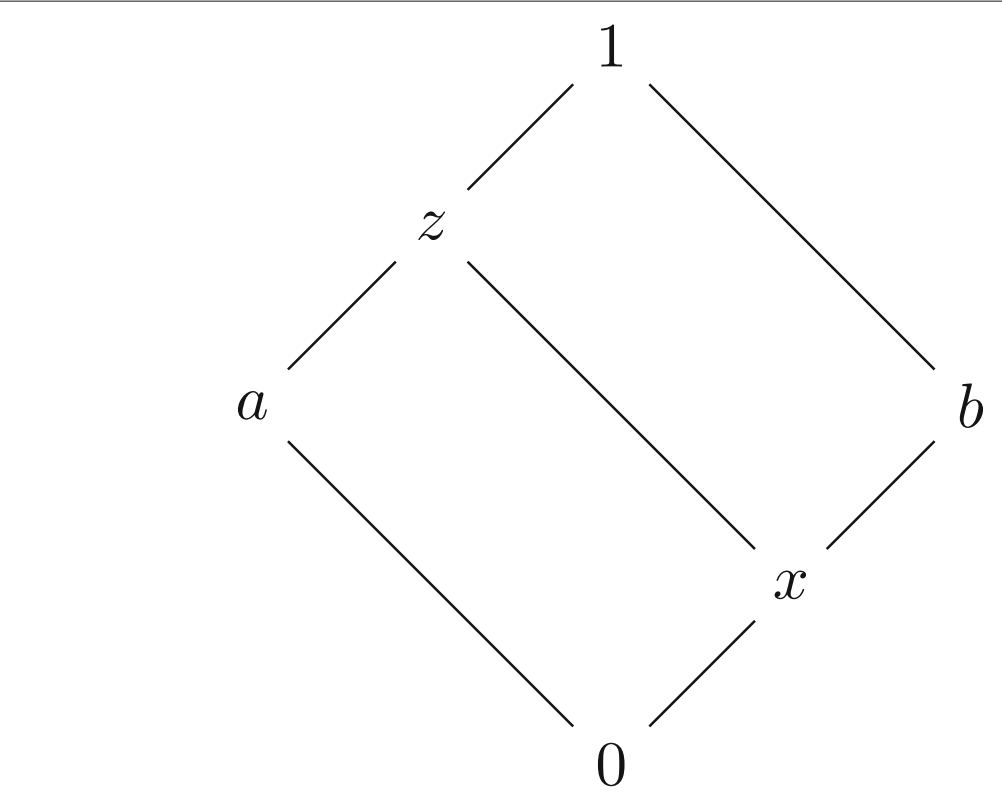

Fig. $3 L_{s}$

Then, $x^{++} \wedge x^{+}=0$ and $x^{\circ \circ} \vee x^{\circ}=1$ imply $x \in L_{S}$. Therefore, $C(L) \subseteq L^{\circ \circ} \cap L_{S}$. Conversely, let $x \in L^{\circ \circ} \cap L_{S}$. Then, $x=x^{\circ \circ}=x^{++}, x^{\circ} \vee x^{\circ \circ}=1$, and $x^{+} \wedge x^{++}=0$. Now,

$$
\begin{aligned}
& x^{\circ} \vee x=x^{\circ} \vee x^{\circ \circ}=1, \\
& x \wedge x^{+}=x^{++} \wedge x^{+}=0 .
\end{aligned}
$$

Thus, $x \in C(L)$, and hence, $L^{\circ \circ} \cap L_{S} \subseteq C(L)$.

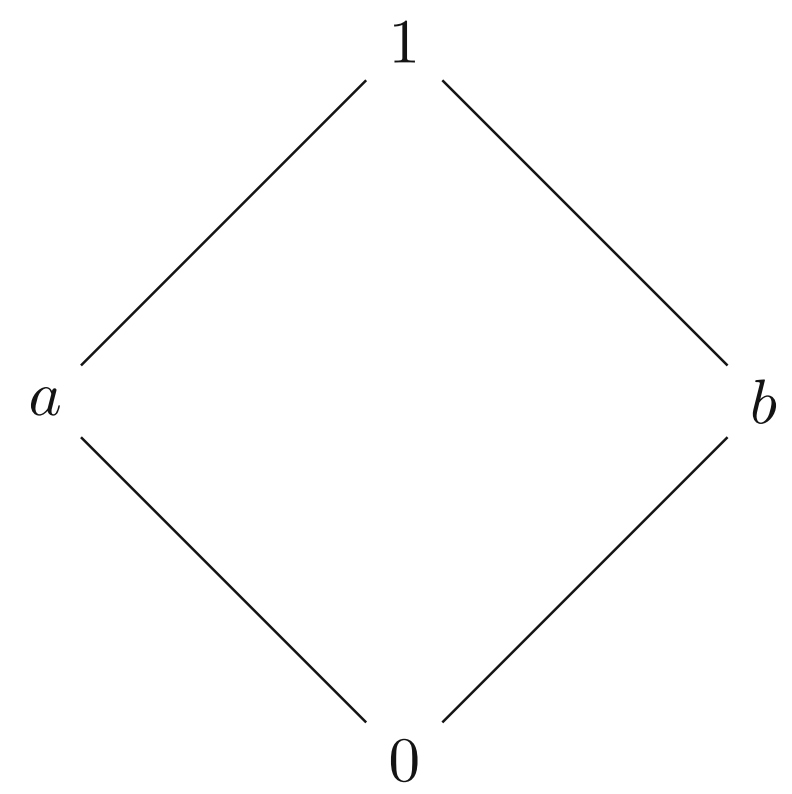

Fig. $4 C(L)$ 
(2) Clearly $0,1 \in C(L)$. Let $a, b \in C(L)$. Then, we have

$$
\begin{aligned}
(a \vee b) \vee(a \vee b)^{\circ} & =a \vee b \vee\left(a^{\circ} \wedge b^{\circ}\right) \text { by Proposition } 1(5) \\
& =\left(a \vee b \vee a^{\circ}\right) \wedge\left(a \vee b \vee b^{\circ}\right) \text { by distributivity of } L \\
& =1 \wedge 1=1, \\
(a \vee b) \wedge(a \vee b)^{+} & =(a \vee b) \wedge\left(a^{+} \wedge b^{+}\right) \text {by Theorem } 1(5) \\
& =\left(a \wedge a^{+} \wedge a^{+}\right) \vee\left(b \wedge a^{+} \wedge b^{+}\right) \text {by distributivity of } L \\
& =0 \vee 0=0 .
\end{aligned}
$$

Then, $a \vee b \in C(L)$. Similarly $a \wedge b \in C(L)$. Therefore, $(C(L) ; \vee, \wedge, 0,1)$ is a bounded sublattice of $L$. Now, we observe that $a^{\circ} \in C(L)$ for all $a \in C(L)$,

$$
\begin{aligned}
a^{\circ \circ} \vee a^{\circ \circ \circ} & =a \vee a^{\circ} \text { as } a^{\circ \circ}=a, \forall a \in C(L) \text { and } a^{\circ \circ \circ}=a^{\circ} \\
& =1, \\
a^{\circ+} \wedge a^{\circ++} & =a^{\circ \circ} \wedge a^{\circ \circ \circ} \text { as } a^{\circ+}=a^{\circ \circ} \\
& =a^{\circ \circ} \wedge a^{\circ} \text { as } a^{\circ \circ}=a^{\circ} \\
& =\left(a^{\circ} \vee a\right)^{\circ}=1^{\circ}=0 \text { by Theorem } 1(5) .
\end{aligned}
$$

Since $a^{\circ}=a^{+}$for all $a \in C(L)$, then ${ }^{\circ}$ coincide with ${ }^{+}$on $C(L)$. Therefore, $\left(C(L), \vee, \wedge,{ }^{\circ}, 0,1\right)$ is a subalgebra of $L$. Since $a \vee a^{\circ}=1$ and $a \wedge a^{\circ}=a^{\circ \circ} \wedge a^{\circ}=\left(a^{\circ} \vee a\right)^{\circ}=$ $1^{\circ}=0$ for all $a \in C(L)$, then $\left(C(L), \vee, \wedge,{ }^{\circ}, 0,1\right)$ is a Boolean subalgebra of $L$. Suppose that $B$ is any Boolean subalgebra of $L$ and $x \in B$. Then, $a \vee a^{\circ}=1$ and $a \wedge a^{+}=a \wedge a^{\circ}=0$. Hence, $a$ is a central element of $L$ and $a \in C(L)$. So, $B \subseteq C(L)$ and $C(L)$ is the greatest Boolean subalgebra of $L$. Using similar agrement, we can show that $C(L)$ is also the greatest Boolean subalgebra of both $L_{S}$ and $L^{\circ \circ}$.

(3) It follows (1) and (2).

The following theorem shows that the centers of isomorphic double $M S$-algebras are isomorphic Boolean algebras.

Theorem 4 If $L$ and $M$ are isomorphic double MS-algebras, then their centers are isomorphic.

Proof Let $h: L \rightarrow M$ be an isomorphism and $a \in C(L)$. Then, $a \vee a^{\circ}=1$ and $a \wedge a^{+}=0$. Hence, $h\left(a \vee a^{\circ}\right)=h(a) \vee h\left(a^{\circ}\right)=h(a) \vee(h(a))^{\circ}=h(1)=1$ and $h(a) \wedge(h(a))^{+}=h(0)=$ 0 . Therefore, $h_{C(L)}(a)=h(a) \in C(M)$. It is clear that $h_{C(L)}$ is an injective $(0,1)$ lattice homomorphism. Let $b \in C(M)$. Then, there exists $b \in L$ such that $b=h(a)=h_{C(L)}(a)$ as $h$ is onto. It follows that $b^{\circ \circ}=(h(a))^{\circ \circ}=h\left(a^{\circ \circ}\right)=h(a)=h_{C(L)}(a)$. Thus, $h_{C(L)}$ is onto. Obviously, $h_{C(L)}$ preserves ${ }^{\circ}$ and ${ }^{+}$. Then, $h_{C(L)}$ is an isomorphism, and hence, $C(L) \cong C(M)$.

For an $M S$-algebra $\left(L,^{\circ}\right)$, it is proved in [3] that $(a]_{L}=\left((a],{ }^{\circ}\right)$ is an $M S$-algebra if and only if $a^{\circ} \in C(L)$, where $(a]=\{x \in L: x \leq a\}=[0, a]$ is a principal ideal of $L$ generated by the element $a$ of $L$, a unary operation ${ }^{\circ} a$ is defined on $(a]$ by $x^{\circ} a=x^{\circ} \wedge a$ for all $x \in(a]$ and $C(L)=\left\{x \in L: x \vee x^{\circ}=1\right\}$ is the center of $L$.

For a double $M S$-algebra $\left(L ;^{\circ},+\right)$, the answer of the following question is given: Under what conditions a principal ideal ( $a], a \in L$ constructs a double $M S$-algebra? 
Theorem 5 Let $L$ be a double MS-algebra. Suppose that a $\in C(L)$, then the relativized algebra $(a]_{L}=\left((a], \wedge, \vee,^{\circ},{ }^{+a}, a, 1\right)$ is a double MS-algebra, where $x^{\circ}=x^{\circ} \wedge$ a and $x^{+a}=x^{+} \wedge a$. Conversely, if $(a]_{L}=\left((a], \wedge, \vee,{ }^{\circ},{ }^{+a}, a, 1\right)$ is a double MS-algebra, then $a \in L_{S}$

Proof Assume that $a \in C(L)$. Hence, $a^{\circ} \in C(L)$. Then, by ([13], Theorem 3.5), $\left((a], \vee, \wedge,{ }^{\circ} a, 0, a\right)$ is an $M S$-algebra, whenever $x^{\circ} a=x^{\circ} \wedge a$. Now, we prove that $((a], \vee, \wedge,+a, 0, a)$ is a dual $M S$-algebra, where $x^{+a}=x^{+} \wedge a$ for any $x \in(a]$. Let $x \in(a]$, we have

$$
\begin{aligned}
x^{+a^{\circ+a} \vee x} & =\left(x^{+} \wedge a\right)^{+a} \vee x \\
& =\left(\left(x^{+} \wedge a\right)^{+} \wedge a\right) \vee x \\
& =\left(\left(x^{++} \vee a^{+}\right) \wedge a\right) \vee x \\
& =\left(x^{++} \wedge a\right) \vee\left(a^{+} \wedge a\right) \vee x \text { by distributivity of } L \\
& =\left(x^{++} \wedge a\right) \vee x \text { as } a^{+} \wedge a=0 \\
& =x \text { as } x \geq x^{++} \geq x^{++} \wedge a .
\end{aligned}
$$

Then, $x \geq x^{+a+a}$. Let $x, y \in(a]$

$$
\begin{aligned}
(x \wedge y)^{+a} & =(x \wedge y)^{\circ} \wedge a \\
& =\left(x^{+} \vee y^{\circ+}\right) \wedge a \\
& =\left(x^{+} \wedge a\right) \vee\left(y^{+} \wedge a\right) \text { by distributivity of } L \\
& =x^{+a} \vee y^{+a}
\end{aligned}
$$

Also, $0^{+a}=a$. Now, for every $x \in(a]$, we have

$$
\begin{aligned}
x^{\circ a+a} & =\left(x^{\circ} \wedge a\right)^{+a} \\
& =\left(x^{\circ} \wedge a\right)^{+} \wedge a \\
& =\left(x^{\circ+} \vee a^{+}\right) \wedge a \\
& =\left(x^{\circ \circ} \vee a^{+}\right) \wedge a \\
& =\left(x^{\circ \circ} \wedge a\right) \vee\left(a^{+} \wedge a\right) \\
& =x^{\circ \circ} \wedge a \text { as } a^{+} \wedge a=0, \\
x^{\circ} a_{a} & =\left(x^{\circ} \wedge a\right)^{\circ} \wedge a \\
& =\left(x^{\circ \circ} \vee a^{\circ}\right) \wedge a \\
& =\left(x^{\circ \circ} \wedge a\right) \vee\left(a^{\circ} \wedge a\right) \text { by distributivity } \\
& =x^{\circ \circ} \wedge a \text { as } a^{+} \wedge a=0 .
\end{aligned}
$$

This deduce that $x^{\circ{ }^{+}+a}=x^{\circ \circ}$. Also, we can get $x^{+a_{a} a}=x^{+a+a}$. Therefore, $(a]_{L}=$ $\left((a], \vee, \wedge,{ }^{\circ},+a, 0, a\right)$ is a double $M S$-algebra.

Conversely, suppose that $a \in L,(a]_{L}=\left((a], \vee, \wedge,{ }^{\circ},{ }^{+}+a, 0\right)$ is a double $M S$-algebra with $x^{\circ a}=x^{\circ} \wedge a$ and $x^{+a}=x^{+} \wedge a$. Since $a$ is the greatest element of $(a]_{L}$, then $a^{+a}=0$ and $a^{\circ} a=0$. This gives $a^{+} \wedge a=0$ and $a^{\circ} \wedge a=0$, respectively. Consequently, $a^{+} \wedge x^{++}=\left(a^{+} \wedge a\right)^{++}=0^{++}=0$ and $a^{\circ \circ} \vee a^{\circ}=\left(a^{\circ} \wedge a\right)^{\circ}=0^{\circ}=1$. Therefore, $a$ is a Stone element of $L$. 
Similarly for the principal filter $[a$ ) of a double $M S$-algebra, we establish the following result, where $[a)=\{x \in L: x \geq a\}=[a, 1]$.

Theorem 6 Let $L$ be a double MS-algebra. If a $\in C(L)$, then the relativized algebra $[a)_{L}=\left([a), \wedge, \vee,{ }^{\circ} a,+a, a, 1\right)$ is a double MS-algebra, where $x^{\circ a}=x^{\circ} \vee a$ and $x^{+a}=x^{+} \vee a$ . Conversely, if $[a)_{L}=\left([a), \wedge, \vee,{ }^{\circ},{ }^{+a}, a, 1\right)$ is a double MS-algebra, then $a \in L_{S}$.

Let $L_{1}, L_{2}$ are double $M S$-algebras. Then, $L_{1} \times L_{2}$ is a double $M S$-algebra, where ${ }^{\circ}$ and ${ }^{+}$ are defined by $(x, y)^{\circ}=\left(x^{\circ}, y^{\circ}\right)$ and $(x, y)^{+}=\left(x^{+}, y^{+}\right)$. Moreover, $\left(L_{1} \times L_{1}\right)^{\circ \circ}=L_{1}^{\circ \circ} \times L_{2}^{\circ \circ}$ and $C\left(L_{1} \times L_{2}\right)=C\left(L_{1}\right) \times C\left(L_{2}\right)$.

As a consequence of Theorem 5 and Theorem 6, we have

Theorem 7 Let $L$ be a double MS-algebra. If $a \in C(L)$, then $\left((a]_{L} \times[a)_{L},{ }^{\circ},+\right)$ is a double MS-algebra, where

$(a]_{L} \times[a)_{L}=\left\{(x, y): x \in(a]_{L}, y \in[a)_{L}\right\}$

and

$(x, y)^{\circ}=\left(x^{\circ} \wedge a, y^{\circ} \vee a\right)$ and $(x, y)^{+}=\left(x^{+} \wedge a, y^{+} \vee a\right)$ for all $(x, y) \in(a]_{L} \times[a)_{L}$.

Now, we introduce the concept of Birkhoff center for a double $M S$-algebra.

Definition 5 An element a of a double MS-algebra L is called a Birkhoff central element if there exist double MS-algebras $L_{1}$ and $L_{2}$ and an isomorphism from $L$ to $L_{1} \times L_{2}$ such that a is mapped to $(1,0)$. The set $B C(L)$ of all Birkhoff central elements of $L$ is called the Birkhoff center.

Theorem 8 Let $L$ be a double MS-algebra. Then, $B C(L)=C(L)$.

Proof Let $a \in B C(L)$. Then, there exist double $M S$-algebras $L_{1}$ and $L_{2}$ and an isomorphism $h$ from $L$ to $L_{1} \times L_{2}$ such that $h(a)=(1,0)$. By Theorem $4, C(L)$ is isomorphic to $C\left(L_{1} \times L_{1}\right)=C\left(L_{1}\right) \times C\left(L_{2}\right)$. Thus, $(1,0) \in C\left(L_{1}\right) \times C\left(L_{2}\right)$. Therefore, $a=h^{-1}(1,0) \in$ $C(L)$ and $B C(L) \subseteq C(L)$.

Conversely, let $a \in C(L)$. Then, by Theorem 5 and Theorem $6, L_{1}=(a]_{L}$ and $L_{2}=[a)_{L}$ are double $M S$-algebras, respectively. The direct product $L_{1} \times L_{2}=(a]_{L} \times[a)_{L}$ is a double $M S$-algebra, by Theorem 7. Notice that $1_{L_{1}}=a$ is the greatest element of $L_{1}$ and $0_{L_{2}}=a$ is the smallest element of $L_{2}$. Define $h: L \rightarrow L_{1} \times L_{2}$ by $h(x)=(a \wedge x, a \vee x)$. It is already seen that $h$ is an isomorphism of $L$ onto $L_{1} \times L_{2}$. Then, $h(a)=(a, a)=\left(1_{L_{1}}, 0_{L_{2}}\right)$ implies $a \in B C(L)$. Therefore, $C(L) \subseteq B C(L)$.

\section{Balanced factor congruences of a double MS-algebra}

In [14], Badawy investigated the relationship between congruences and de Morgan filters of decomposable $M S$-algebras. In this section, we study the connection between congruences and central elements of a double $M S$-algebra.

Let $a$ be an element of a double $M S$-algebra $L$. Define a binary relation $\theta_{a}$ on $L$ by

$(x, y) \in \theta_{a}$ iff $x \vee a=y \vee a$.

Proposition 3 For any two elements $a$ and $b$ of a double MS-algebra L, we have (1) $\theta_{a}$ is a lattice congruence on $L$ with $\operatorname{Ker} \theta_{a}=(a]$, 
(2) $a \leq b$ iff $\theta_{a} \subseteq \theta_{b}$,

(3) $a=b$ iff $\theta_{a}=\theta_{b}$,

(4) $\theta_{0}=\Delta$ and $\theta_{1}=\nabla$,

(5) $\theta_{a}$ is the smallest lattice congruence containing $(0, a)$.

Proof (1). Obviously $\theta_{a}$ is an equivalence relation on $L$. Let $(x, y) \in \theta_{a}$. Then, $x \vee a=y \vee a$. For all $z \in L$, by associativity and commutativity of $\vee$, we have

$$
\begin{aligned}
(x \vee z) \vee a & =x \vee(z \vee a) \\
& =x \vee(a \vee z) \\
& =(x \vee a) \vee z \\
& =y \vee(a \vee z \\
& =y \vee(z \vee a) \\
& =(y \vee z) \vee a,
\end{aligned}
$$

and

$$
\begin{aligned}
(x \wedge z) \vee a & =(x \vee a) \wedge(z \vee a) \text { by distributivity of } L \\
& =(y \vee a) \wedge(z \vee a) \\
& =(y \wedge z) \vee a \text { by distributivity of } L .
\end{aligned}
$$

Then, by Theorem $2, \theta_{a}$ is a lattice congruence on $L$. Now

$$
\begin{aligned}
\operatorname{Ker} \theta_{a} & =\left\{x \in L:(0, x) \in \theta_{a}\right\} \\
& =\{x \in L: a=0 \vee a=x \vee a\} \\
& =\{x \in L: x \leq a\}=[a) .
\end{aligned}
$$

(2) Let $a \leq b$ and $(x, y) \in \theta_{a}$. Hence, $x \vee a=y \vee a$. Then, $x \vee a \vee b=y \vee a \vee b$ implies $x \vee b=y \vee b$. This gives $(x, y) \in \theta_{a}$ and $\theta_{a} \subseteq \theta_{b}$. Conversely, let $\theta_{a} \subseteq \theta_{b}$. Since $(a \wedge b) \vee a=a=a \vee a$, then $(a \wedge b, a) \in \theta_{a}$. By hypotheses, $(a \wedge b, a) \in \theta_{b}$. Then, $(a \wedge b) \vee b=a \vee b$ implies $b=a \vee b$. Therefore, $a \leq b$.

(3) It is obvious.

(4) Since for any $(x, y) \in \theta_{0}$, we have $x=y$. Then, $\theta_{0}=\Delta$. For all $x, y \in L$, we have $x \vee 1=1=y \vee 1$ and hence $(x, y) \in \theta_{1}$. Hence, $\theta_{1}=\nabla$.

(5) Let $\theta$ be a lattice congruence containing $(0, a)$. Suppose that $(x, y) \in \theta_{a}$. Then, $x \vee a=$ $y \vee a$. Since $(x, x),(0, a) \in \theta$, then $(x, x \vee a) \in \theta$. Also, $(y, y),(0, a) \in \theta$ give $(y, y \vee a) \in \theta$. Then, $(x, x \vee a),(x \vee a, y) \in \theta$ imply $(x, y) \in \theta$. So, $\theta_{a} \subseteq \theta$.

Proposition 4 For any two elements $a$ and $b$ of a double MS-algebra $L$, we have
(1) $\theta_{a \wedge b}=\theta_{a} \cap \theta_{b}$,
(2) $\theta_{a \vee b}=\theta_{a} \vee \theta_{b}$
(3) $\theta_{a} \circ \theta_{b}=\theta_{b} \circ \theta_{a}$
(4) $\theta_{a} \circ \theta_{b}=\theta_{a} \vee \theta_{b}$ 
Proof (1). Since $a \wedge b \leq a, b$, then by Proposition 3(2), $\theta_{a \wedge b} \subseteq \theta_{a}, \theta_{b}$. Thus, $\theta_{a \wedge b} \subseteq \theta_{a} \cap \theta_{b}$. Conversely, let $(x, y) \in \theta_{a} \cap \theta_{b}$. Then

$$
\begin{aligned}
(x, y) \in \theta_{a} \cap \theta_{b} & \Rightarrow(x, y) \in \theta_{a} \text { and }(x, y) \in \theta_{b} \\
& \Rightarrow x \vee a=y \vee a \text { and } x \vee b=y \vee b \\
& \Rightarrow(x \vee a) \wedge(x \vee b)=(y \vee a) \wedge(y \vee b) \\
& \Rightarrow x \vee(a \wedge b)=y \vee(a \wedge b) \text { by distributivity of } L \\
& \Rightarrow(x, y) \in \theta_{a \wedge b}
\end{aligned}
$$

Therefore, $\theta_{a} \cap \theta_{b} \subseteq \theta_{a \wedge b}$ and $\theta_{a \wedge b}=\theta_{a} \cap \theta_{b}$.

(2) Since $a, b \leq a \vee b$, then $\theta_{a}, \theta_{b} \subseteq \theta_{a \vee b}$. Hence, $\theta_{a \vee b}$ is an upper bound of $\theta_{a}$ and $\theta_{b}$. Assume that $\theta_{c}$ is an upper bound of $\theta_{a}$ and $\theta_{b}$. Then, by Proposition 3(2), $\theta_{a}, \theta_{b} \subseteq \theta_{c}$ imply that $a, b \leq c$. We prove that $\theta_{a \vee b} \subseteq \theta_{c}$. Let $(x, y) \in \theta_{a \vee b}$. Then, $x \vee a \vee b=y \vee a \vee b$. Hence, $x \vee a \vee b \vee c=y \vee a \vee b \vee c$ implies $x \vee c=y \vee c$ and $(x, y) \in \theta_{c}$. This shows that $\theta_{a \vee b}$ is the least upper bound of $\theta_{a}$ and $\theta_{b}$, that is, $\theta_{a \vee b}=\theta_{a} \vee \theta_{b}$.

(3) Let $(x, y) \in \theta_{a} \circ \theta_{b}$. Then, there exists $q \in L$ such that $(x, q) \in \theta_{a}$ and $(q, y) \in \theta_{b}$. Thus, $x \vee a=q \vee a$ and $q \vee b=y \vee b$. Put $s=(a \vee y) \wedge(b \vee x)$. Now

$$
\begin{aligned}
a \vee s & =a \vee\{(a \vee y) \wedge(b \vee x)\} \\
& =(a \vee a \vee y) \wedge(a \vee b \vee x) \text { by distributivity of } L \\
& =(a \vee y) \wedge(a \vee b \vee q) \text { as } a \vee q=a \vee x \\
& =(a \vee y) \wedge(a \vee b \vee y) \text { as } b \vee q=b \vee y \\
& =a \vee\{y \wedge(b \vee y)\} \text { by distributivity of } L \\
& =a \vee y \text { by the absorbtion identity. }
\end{aligned}
$$

Then, $(s, y) \in \theta_{a}$. Also

$$
\begin{aligned}
b \vee s & =b \vee\{(a \vee y) \wedge(b \vee x)\} \\
& =(a \vee b \vee y) \wedge(b \vee x) \text { by distributivity of } L \\
& =(b \vee a \vee q) \wedge(b \vee x) \text { as } b \vee q=b \vee y \\
& =(b \vee a \vee x) \wedge(b \vee x) \text { as } x \vee a=q \vee a \\
& =b \vee\{(a \vee x) \wedge x\} \text { by distributivity of } L \\
& =b \vee x \text { by the absorbtion identity. }
\end{aligned}
$$

Then, $(x, s) \in \theta_{b}$. Therefore, $(x, y) \in \theta_{b} \circ \theta_{a}$ and $\theta_{a} \circ \theta_{b} \subseteq \theta_{b} \circ \theta_{a}$. Conversely, let $(x, y) \in$ $\theta_{b} \circ \theta_{a}$. Then, there exists $s \in L$ such that $(x, s) \in \theta_{b}$ and $(s, y) \in \theta_{a}$. Set $t=(b \vee y) \wedge(a \vee x)$. Then, we can get $a \vee t=a \vee x$ and $b \vee t=b \vee y$ which means $(x, t) \in \theta_{a}$ and $(t, y) \in \theta_{b}$. Therefore, $(x, y) \in \theta_{a} \circ \theta_{b}$. So, $\theta_{b} \circ \theta_{a} \subseteq \theta_{a} \circ \theta_{b}$.

(4) Let $(x, y) \in \theta_{a} \circ \theta_{b}$. Then, there exists $q \in L$ such that $(x, q) \in \theta_{a}$ and $(q, y) \in \theta_{b}$. Then, $x \vee a=q \vee a$ and $q \vee b=y \vee b$. Using associativity and commutativity of $\vee$, we get

$$
(a \vee b) \vee x=(a \vee x) \vee b=(a \vee q) \vee b=a \vee(q \vee b)=a \vee(y \vee b)=(a \vee b) \vee y
$$


Then, $(x, y) \in \theta_{a \vee b}$. Conversely, let $(x, y) \in \theta_{a \vee b}$. Then, $a \vee b \vee x=a \vee b \vee y$. Set $q=(a \vee x) \wedge(b \vee y)$. We have

$$
\begin{aligned}
a \vee q & =a \vee\{(a \vee x) \wedge(b \vee y)\} \\
& =(a \vee x) \wedge(a \vee b \vee y) \text { by distributivity of } L \\
& =(a \vee x) \wedge(a \vee b \vee x) \\
& =a \vee x \text { as } a \vee x \leq a \vee b \vee x .
\end{aligned}
$$

Then, $(x, q) \in \theta_{a}$. Also, we can get $(q, y) \in \theta_{b}$. Therefore, $(x, y) \in \theta_{a} \circ \theta_{b}$ and $\theta_{a \vee b} \subseteq \theta_{a} \circ \theta_{b}$.

Theorem 9 For any two elements $a$ and $b$ of a double MS-algebra $L$, we have

(1) $\theta_{a}$ is compatible with ${ }^{\circ}$ if and only if $a \vee a^{\circ}=1$,

(2) $\theta_{a}$ is compatible with ${ }^{+}$if and only if $a^{+} \wedge a^{++}=0$,

(3) $\theta_{a}$ is a congruence on $L$ if and only if $a \in C(L)$.

Proof (1). Let $(x, y) \in \theta_{a}$ and $a^{\circ} \vee a=1$. Then, $x \vee a=y \vee a$. We prove that $(x, y) \in \theta_{a}$ implies $\left(x^{\circ}, y^{\circ}\right) \in \theta_{a}$.

$$
\begin{aligned}
(x, y) \in \theta_{a} & \Rightarrow x \vee a=y \vee a \\
& \Rightarrow x^{\circ} \wedge a^{\circ}=(x \vee a)^{\circ}=(y \vee a)^{\circ}=y^{\circ} \wedge a^{\circ} \text { by Theorem 1(5) } \\
& \Rightarrow\left(x^{\circ} \wedge a^{\circ}\right) \vee a=\left(y^{\circ} \wedge a^{\circ}\right) \vee a \text { by joining two sides with } a \\
& \Rightarrow\left(x^{\circ} \vee a\right) \wedge\left(a^{\circ} \vee a\right)=\left(x^{\circ} \vee a\right) \wedge\left(a^{\circ} \vee a\right) \text { by the distributivity of } L \\
& \Rightarrow x^{\circ} \vee a=x^{\circ} \vee a \text { as } a^{\circ} \vee a=1 \\
& \Rightarrow\left(x^{\circ}, y^{\circ}\right) \in \theta_{a}
\end{aligned}
$$

Then, $\left(x^{\circ}, y^{\circ}\right) \in \theta_{a}$. Conversely, let $\theta_{a}$ is compatible with ${ }^{\circ}$. Since $(0, a) \in \theta_{a}$ by Proposition $3(5)$, then $\left.\left(1, a^{\circ}\right)\right) \in \theta_{a}$. Hence, $\left.(a, a),\left(1, a^{\circ}\right)\right) \in \theta_{a}$ implies $\left(1, a \vee a^{\circ}\right) \in \theta_{a}$. Therefore, $1=1 \vee a=a \vee\left(a \vee a^{\circ}\right)=a \vee b$.

(2) Let $a^{+} \wedge a^{++}=0$. Using the properties of dual MS-algebra $\left(L ;^{+}\right)$and Proposition 1, we get $a^{+} \vee a \geq a^{+} \vee a^{++}=\left(a^{+} \wedge a^{++}\right)^{+}=0^{+}=1$ and hence $a^{+} \vee a=1$. Now, let $(x, y) \in \theta_{a}$. We have

$$
\begin{aligned}
(x, y) \in \theta_{a} & \Rightarrow x \vee a=y \vee a \\
& \Rightarrow x^{+} \wedge a^{+}=(x \vee a)^{+}=(y \vee a)^{+}=y^{+} \wedge a^{+} \text {by Proposition 1(5) } \\
& \Rightarrow\left(x^{+} \wedge a^{+}\right) \vee a=\left(y^{+} \wedge a^{+}\right) \vee a \text { by joining two sides with } a \\
& \Rightarrow\left(x^{+} \vee a\right) \wedge\left(a^{+} \vee a\right)=\left(x^{+} \vee a\right) \wedge\left(a^{+} \vee a\right) \text { by the distributivity of } L \\
& \Rightarrow x^{+} \vee a=x^{+} \vee a \text { as } a^{+} \vee a=1 .
\end{aligned}
$$

Then, $\left(x^{+}, y^{+}\right) \in \theta_{a}$. Conversely, let $\theta_{a}$ is compatible with ${ }^{+}$. Then, $(0, a) \in \theta_{a}$ implies $\left.\left(1, a^{+}\right)\right) \in \theta_{a}$. Since $\left.(a, a),\left(1, a^{+}\right)\right) \in \theta_{a}$, then $\left(1, a \vee a^{+}\right) \in \theta_{a}$. Hence, $1=1 \vee a=a \vee a^{+}$. It follows that $a^{+} \wedge a^{++}=\left(a \vee a^{+}\right)^{+}=1^{+}=0$.

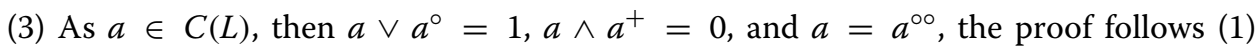
and (2).

Now, we introduce the concept of factor congruences for double $M S$-algebras. 
Definition 6 A congruence $\theta$ on a double MS-algebra $L$ is called a factor congruence if there is a congruence $\psi$ on $L$ such that $\theta \wedge \psi=\Delta, \theta \vee \psi=\nabla$ and $\theta$ permutes with $\psi$.

Theorem 10 Let $L$ be a double MS-algebra and $\theta$ a congruence on $L$. Then, $\theta$ is a factor congruence on $L$ if and only if $\theta=\theta_{a}$ for some $a \in C(L)$.

Proof Let $a \in C(L)$. Hence, $a^{\circ} \in C(L)$. Using Theorem 9(3), we deduce that $\theta_{a}$ and $\theta_{a^{\circ}}$ are congruences on $L$. Hence, we get

$$
\begin{aligned}
\theta_{a} \vee \theta_{a^{\circ}} & =\theta_{a \vee a^{\circ}} \text { by Proposition } 4(2) \\
& =\theta_{1} \text { as } a \vee a^{\circ}=1 \\
& =\nabla \text { by Proposition 3(4), } \\
\theta_{a} \cap \theta_{a^{\circ}} & =\theta_{a \wedge a^{\circ}} \text { by Proposition 4(1) } \\
& =\theta_{0} \text { as } a \wedge a^{\circ}=0 \\
& =\Delta \text { by Proposition 3(4), } \\
\theta_{a} \circ \theta_{a^{\circ}} & =\theta_{a^{\circ}} \circ \theta_{a} \text { by Proposition 4(3). }
\end{aligned}
$$

Therefore, $\theta_{a}$ is a factor congruence on $L$, whenever $a \in C(L)$. Conversely, let $\theta$ be a factor congruence on $L$. Then, there exists a congruence $\psi$ on $L$ such that $\theta \vee \psi=\nabla$ and $\theta \cap \psi=\Delta$. Since $(0,1) \in \nabla=\theta \vee \psi=\theta \circ \psi$, then there exists $x \in L$ such that $(0, x) \in \theta$ and $(x, 1) \in \psi$. Thus, $\left(0, x^{\circ \circ}\right) \in \theta$ and $\left(x^{\circ \circ}, 1\right) \in \psi$. We prove that $\theta=\theta_{x^{\circ \circ}}$ such that $x^{\circ 0} \in C(L)$. Since $\left(0, x^{\circ \circ}\right) \in \theta$, then by Proposition 3(5), $\theta_{x^{\circ \circ}} \subseteq \theta$. Now, let $(p, q) \in \theta$. Then, $(p, q),\left(x^{\circ \circ}, x^{\circ \circ}\right) \in \theta$ implies $\left(p \vee x^{\circ \circ}, q \vee x^{\circ \circ}\right) \in \theta$. Since $\left(x^{\circ \circ}, 1\right),(p, p),(q, q) \in \psi$, then $\left(x^{\circ \circ} \vee p, 1\right),\left(x^{\circ \circ} \vee q, 1\right) \in \psi$. Hence, $\left(x^{\circ \circ} \vee p, x^{\circ \circ} \vee q\right) \in \psi$. Therefore, $\left(x^{\circ \circ} \vee p, x^{\circ \circ} \vee q\right) \in$ $\theta \cap \psi=\Delta$. It follows that $x^{\circ \circ} \vee p=x^{\circ \circ} \vee q$ and hence $(p, q) \in \theta_{x^{\circ \circ}}$. So, $\theta \subseteq \theta_{\circ \circ}$ and $\theta=\theta_{x^{\circ \circ}}$. This deduce that $\theta_{x^{\circ \circ}}$ is a congruence on $L$. So, by Theorem 9(3), $x^{\circ \circ} \in C(L)$.

Now, we introduce the concept of balanced factor congruences of a double $M S$-algebra.

Definition 7 A congruence $\theta$ on a double MS-algebra $L$ is called balanced if $(\theta \vee \alpha) \cap$ $\left(\theta \vee \alpha^{\prime}\right)=\theta$ for all factor congruence $\alpha$ and its complement $\alpha$. The set $\mathbf{B}(L)$ of all balanced factor congruences which admit a balanced complement is called the Boolean center of $L$.

Example 2 Consider the double MS-algebra $L$ as in Example 1. Factor congruences on $L$ are given as follows:

$$
\theta_{0}=\Delta, \theta_{1}=\nabla, \theta_{a}=\{\{0, c, a\},\{x, y, z\},\{b, d, 1\}\}, \theta_{b}=\{\{0, x, b\},\{c, y, d\},\{a, z, 1\}\} .
$$

It is observed that the Boolean lattice $\mathbf{B}(L)$, of all balanced factor congruences is $\mathbf{B}(L)=$ $\left\{\theta_{0}, \theta_{a}, \theta_{b}, \theta_{1}\right\}$ which is represented in Fig. 5. Clearly $C(L)$ and $\mathbf{B}(L)$ are isomorphic Boolean lattices.

Lemma 1 Let $L$ be a double MS-algebra and $x \in C(L)$. Then, $\theta_{x}$ is balanced.

Proof Let $\alpha$ be a factor congruence on $L$ and $\alpha$ be its complement. Using Theorem 10, there exist $a, b \in C(L)$ such that $\alpha=\theta_{a}$ and $\dot{\alpha}=\theta_{b}$. Hence, $\alpha \cap \dot{\alpha}=\Delta$ and $\alpha \vee \dot{\alpha}=\nabla$. 


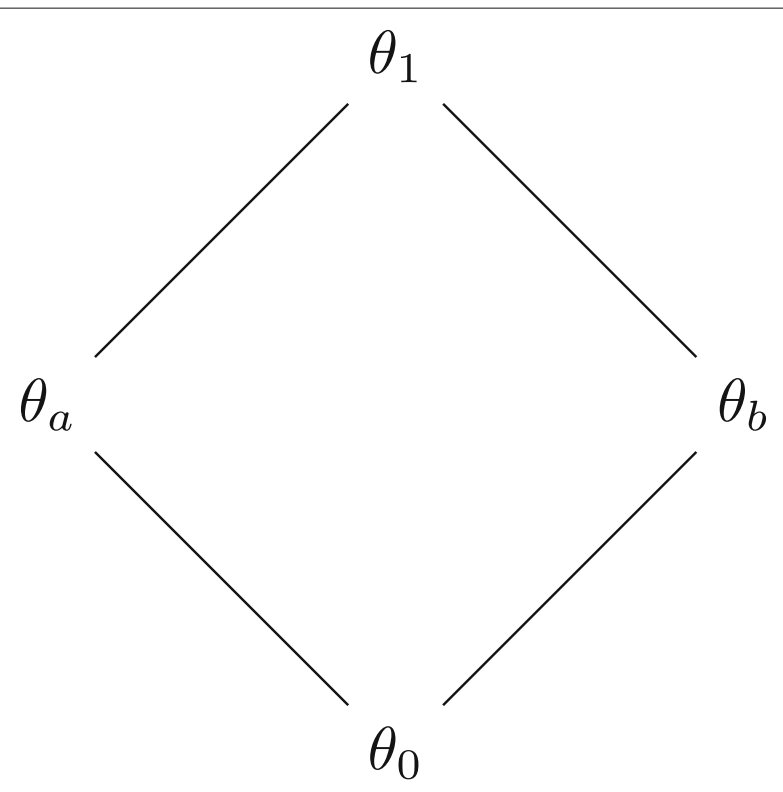

Fig. 5 B $(L)$

We have

$$
\begin{aligned}
\left(\theta_{x} \vee \alpha\right) \cap\left(\theta_{x} \vee \alpha\right) & =\left(\theta_{x} \vee \theta_{a}\right) \cap\left(\theta_{x} \vee \theta_{b}\right) \\
& =\theta_{x \vee a} \cap \theta_{x \vee b} \text { by Proposition 4(2) } \\
& =\theta_{(x \vee a) \wedge(x \vee b)} \text { by Proposition 4(1) } \\
& =\theta_{x \vee(a \wedge b)} \text { by distributivity of } L \\
& =\theta_{x} \vee\left(\theta_{a} \cap \theta_{b}\right) \text { by Proposition } 4(2) \text { and (1), respectively } \\
& =\theta_{x} \vee\left(\alpha \cap \alpha^{\prime}\right) \\
& =\theta_{x} \vee \Delta \text { as } \alpha \cap \dot{\alpha}=\Delta \\
& =\theta_{x} \text { as } \Delta \subseteq \theta_{x} \text { for all } x \in L .
\end{aligned}
$$

Then, $\theta_{x}$ is balanced.

We close this section with the following two important results.

Theorem 11 Let $L$ be a double MS-algebra. Then, the Boolean center $\mathbf{B}(L)$ of $L$ is precisely the $\operatorname{set}\left\{\theta_{a}: a \in C(L)\right\}$.

Theorem 12 Let L be a double MS-algebra. Then, the Boolean center $\mathbf{B}(L)$ is a Boolean algebra and the mapping $a \mapsto \theta_{a}$ is an isomorphism of $C(L)$ onto $\mathbf{B}(L)$.

Proof The set of all balanced factor congruences of $L$ is $\mathbf{B}(L)=\left\{\theta_{a}: a \in C(L)\right\}$ by Theorem 11. It is clear that $\theta_{1}=\nabla$ is the greatest element of $\mathbf{B}(L)$ and $\theta_{0}=\Delta$ is the smallest element of $\mathbf{B}(L)$ by Proposition 3(4). Also, by Proposition 4(1),(2), respectively, we have $\theta_{a} \cap \theta_{b}=\theta_{a \wedge b}$ and $\theta_{a} \vee \theta_{b}=\theta_{a \vee b}$ for all $\theta_{a}, \theta_{b} \in \mathbf{B}(L)$. Then, $\left(\mathbf{B}(L) ; \cap, \vee, \theta_{0}, \theta_{1}\right)$ is a bounded lattice. For all $\theta_{a}, \theta_{b}, \theta_{c} \in \mathbf{B}(L)$, by distributivity of $C(L)$, we get $\theta_{a} \cap\left(\theta_{b} \vee \theta_{c}\right)=$ $\theta_{a} \cap \theta_{b \vee c}=\theta_{a \wedge(a \vee b)}=\theta_{(a \vee b) \wedge(a \vee c)}=\theta_{a \vee b} \cap \theta_{a \vee c}=\left(\theta_{a} \vee \theta_{b}\right) \cap\left(\theta_{a} \vee \theta_{c}\right)$. Thus, $\mathbf{B}(L)$ is 
a distributive lattice. The complement of $\theta_{a}$ is $\theta_{a^{\circ}}$. Then, $\mathbf{B}(L)$ is a Boolean algebra. The proof of the rest part of this theorem is straightforward.

Acknowledgements

We thank the referees for valuable comments and suggestions for improving the paper.

Authors' contributions

The author read and approved the final manuscript.

Competing interests

The author declares that he has no competing interests.

\section{Publisher's Note}

Springer Nature remains neutral with regard to jurisdictional claims in published maps and institutional affiliations.

Received: 26 May 2018 Accepted: 1 September 2018

Published online: 24 April 2019

\section{References}

1. Blyth, T. S, Varlet, J. C: On a common abstraction of de Morgan algebras and Stone algebras. Proc. Roy. Soc. Edinburgh. 94A, 301-308 (1983)

2. Blyth, T. S, Varlet, J. C: Subvarieties of the class of MS-algebras. Proc. Roy. Soc. Edinb. 95 A, 157-167 (1983)

3. Badawy, A, Guffova, D, Haviar, M: Triple construction of decomposable MS-algebras. Acta Univ. Palacki. Olomuc. Fac. Rer. Nat. Math. 51(2), 53-65 (2012)

4. Badawy, A: $d_{L}$-Filters of principal $M S$-algebras. J. Egypt. Math. Soc. 23, 463-469 (2015)

5. Badawy, A, El-Fawal, R: Homomorphism and subalgebras of decomposable $M S$-algebras. J. Egypt. Math. Soc. 25, 119-124 (2017)

6. Blyth, T. S, Varlet, J. C: Double MS-algebras. Proc. Roy. Soc. Edinb. 94, 157-169 (1984)

7. Congwen, L: The class of double MS-algebras satisfying the complement property. Bull. Soc. Sci. Liège. 70(1), 51-59 (2001)

8. Haviar, M: Affine complet algebras abstracting double Stone and Kleene algebras. Acta Univ. M. Belii. Math. 4, 39-52 (1996)

9. Wang, L: Congruence pairs of algebras abstracting double Kleene and Stone algebras. Chamchuri J. of Math. 5, 1-9 (2013)

10. Badawy, A: Regular double $M S$-algebras. Appl. Math. Inf. Sci. 11(2), 115-122 (2017)

11. Blyth, T. S, Varlet, J. C: Ockham Algebras (1994)

12. Blyth, T. S: Lattices and ordered algebric structures (2005)

13. Badawy, A, Atallah, M: $M S$-intervals of an $M S$-algebra. Hacet. J. of Math. and Stat (2019). in press

14. Badawy, A.: Congruences and de Morgan filters of decomposable $M$ S-algebras. SE Asian Bull. Math. 43, 13-25 (2019)

\section{Submit your manuscript to a SpringerOpen ${ }^{\circ}$ journal and benefit from:}

- Convenient online submission

Rigorous peer review

- Open access: articles freely available online

- High visibility within the field

- Retaining the copyright to your article

Submit your next manuscript at $\boldsymbol{\triangleright}$ springeropen.com 